\title{
Contribution of comorbidities to functional impairment is higher in heart failure with preserved than with reduced ejection fraction
}

\author{
Frank Edelmann • Raoul Stahrenberg • Götz Gelbrich • Kathleen Durstewitz • Christiane E. Angermann • \\ Hans-Dirk Düngen · Thomas Scheffold • Christian Zugck • Bernhard Maisch • Vera Regitz-Zagrosek • \\ Gerd Hasenfuß $\cdot$ Burkert M. Pieske $\cdot$ Rolf Wachter
}

Received: 2 November 2010/ Accepted: 7 March 2011/Published online: 17 March 2011

(C) The Author(s) 2011. This article is published with open access at Springerlink.com

\begin{abstract}
Background Comorbidities negatively affect prognosis more strongly in heart failure with preserved (HFpEF) than with reduced (HFrEF) ejection fraction. Their comparative impact on physical impairment in HFpEF and HFrEF has not been evaluated so far.

Methods and results The frequency of 12 comorbidities and their impact on NYHA class and SF-36 physical functioning score (SF-36 PF) were evaluated in 1,294 patients with HFpEF and 2,785 with HFrEF. HFpEF patients had lower NYHA class $(2.0 \pm 0.6$ vs. $2.4 \pm 0.6, p<0.001)$ and higher SF-36PF score ( $54.4 \pm 28.3$ vs. $54.4 \pm 27.7, p<0.001)$. All comorbidities were significantly $(p<0.05)$ more frequent in
\end{abstract}

F. Edelmann and R. Stahrenberg contributed equally to this work.

F. Edelmann $(\bowtie) \cdot$ R. Stahrenberg $\cdot$ K. Durstewitz .

G. Hasenfuß - R. Wachter

Department of Cardiology and Pneumology,

University of Göttingen, 37075 Göttingen, Germany

e-mail: fedelmann@med.uni-goettingen.de

G. Gelbrich

Coordination Center for Clinical Trials,

University of Leipzig, Leipzig, Germany

C. E. Angermann

Department of Internal Medicine I,

Center for Cardiovascular Medicine,

University of Würzburg, Würzburg, Germany

H.-D. Düngen

Department of Cardiology,

Charité-Universitätsmedizin Berlin, Berlin, Germany

T. Scheffold

Institute for Heart and Circulation Research,

University Witten/Herdecke, Dortmund, Germany
HFrEF, except hypertension and obesity, which were more frequent in $\operatorname{HFpEF}(p<0.001)$. Adjusting for age and gender, COPD, anemia, hyperuricemia, atrial fibrillation, renal dysfunction, cerebrovascular disease and diabetes had a similar ( $p$ for interaction $>0.05$ ) negative effect in both groups. Obesity, coronary artery disease and peripheral arterial occlusive disease exerted a significantly $(p<0.05)$ more adverse effect in HFpEF, while hypertension and hyperlipidemia were associated with fewer $(p<0.05)$ symptoms in HFrEF only. The total impact of comorbidities on NYHA (AUC for prediction of NYHA III/IV vs. I/II) and SF-36 PF $\left(r^{2}\right)$ in multivariate analyses was approximately 1.5 -fold higher in HFpEF, and also much stronger than the impact of a

\author{
C. Zugck \\ Department of Cardiology, \\ University Hospital Heidelberg, Heidelberg, Germany \\ B. Maisch \\ Department of Cardiology, \\ University Hospital Giessen and Marburg, \\ Marburg, Germany \\ V. Regitz-Zagrosek \\ Center for Cardiovascular Research, \\ Charité University Medicine, Berlin, Germany \\ B. M. Pieske \\ Department of Cardiology, \\ Medical University Graz, Graz, Austria
}


$10 \%$ decrease in ejection fraction in $\mathrm{HFrEF}$ or a $5 \mathrm{~mm}$ decrease in left ventricular end-diastolic diameter in HFpEF. Conclusion The impact of comorbidities on physical impairment is higher in HFpEF than in HFrEF. This should be considered in the differential diagnosis and in the treatment of patients with $\mathrm{HFpEF}$.

Keywords Heart failure with preserved ejection fraction . Heart failure with reduced ejection fraction .

Comorbidities · Physical impairment

\section{Introduction}

Congestive heart failure (CHF) is a prevalent disease and a heavy burden for health-care systems [1] as well as for the affected individual. The leading symptom is an impairment of exercise performance, limiting patients' functioning and quality of daily life. However, physical limitations in individuals with $\mathrm{CHF}$ are only partly explained by cardiac function [2]. Comorbidities are prevalent in CHF [3] and, with the aging of Western populations, will be even more so in the future. They are known to strongly influence the overall prognosis and health-care utilization [4]. However, data are limited regarding the influence of comorbidities on signs and symptoms in CHF. Furthermore, most analyses dealing with the impact of comorbidities evaluated the influence of one single disease entity at a time. Dyspnea and a limitation in exercise capacity are usually the complaints in $\mathrm{CHF}$ [5] that induce a specialist consultation to confirm a suspicion of CHF. They are the most important subjective parameters to evaluate the course of the disease in an individual. More than $50 \%$ of patients with the clinical syndrome of heart failure have a normal left ventricular ejection fraction [6, 7]. These cases can therefore be termed "heart failure with preserved ejection fraction" (or HFpEF) as compared to "heart failure with reduced ejection fraction" (or HFrEF). Although the overall prognosis in $\mathrm{HFpEF}$ is similarly grim as in HFrEF [6, 7], patients frequently die from non-cardiac causes and less often from heart failure than HFrEF patients [8-10]. Comorbidities are therefore believed to play a more important role in $\mathrm{HFpEF}$ than in $\mathrm{HFrEF}$ [11]. Still, knowledge about the impact of such comorbidities on symptoms in HFpEF is even more limited than in HFrEF.

We therefore aimed to compare the frequency of 12 major comorbidities and their impact on NYHA class and SF-36 physical functioning (PF) score in a large nationwide sample of $\mathrm{CHF}$ patients. We hypothesized that the impact of comorbidities would be higher in patients with HFpEF than with HFrEF.

\section{Methods}

Study design and setting

We performed a retrospective cross-sectional analysis of all patients who were screened for clinical trials performed within the framework of the German Competence Network Heart Failure (CNHF).

The CNHF is a government-sponsored nation-wide network consisting of infrastructure and scientific projects dedicated to improvement in efficiency and efficacy of research in $\mathrm{CHF}$, quality of care for $\mathrm{CHF}$ patients, and educating the public about CHF (for details see Mehrhof et al. [12.] and (www.ghfn.eu)). All trials within the CNHF comply with the declaration of Helsinki; the protocols were approved by the responsible ethics committees and all patients gave written informed consent. Diagnostic procedures within $\mathrm{CNHF}$ were performed according to standard operating procedures (SOP). For all clinical projects within the framework of CNHF, a harmonized basic clinical data set consisting of 190 items has been developed including echocardiographic parameters. Echocardiography was performed according to international guidelines transferred into CNHF SOPs. Data storage and analysis were performed at a central data management unit.

Within the basic data set, information was available on the following 12 major comorbidities that are typical for an elderly heart failure population [4]: presence of coronary artery disease (CAD), peripheral arterial occlusive disease (PAOD) and cerebrovascular disease (i.e., history of stroke or transitory ischemic attack). These were classified according to medical history. Chronic obstructive pulmonary disease (COPD), diabetes mellitus, hypertension, hyperlipidemia and hyperuricemia were classified according to medical history or respective medication. Renal dysfunction (i.e., eGFR $<60 \mathrm{ml} / \mathrm{min}$ per $\mathrm{m}^{2}$; eGFR calculated by MDRD formula [13]) and anemia (i.e., $\mathrm{Hb}<12$ or $13 \mathrm{~g} / \mathrm{dl}$ for women or men, respectively) were classified according to history and pathological laboratory values at screening. Obesity was defined as a body mass index $>30 \mathrm{~kg} / \mathrm{m}^{2}$; diagnosis of atrial fibrillation was based on baseline ECGs and therefore represents persistent or permanent atrial fibrillation only. NYHA functional class was assessed by screening physicians and quality of life by the SF-36 standard questionnaire [14].

\section{Patients}

Data were assessed at the individual patient level at the screening visit for each trial and were pooled for this analysis across five interventional and six non-interventional trials (for details see Mehrhof et al. [12]). Patients were classified as having CHF by experienced cardiologists 
at screening, and as having HFpEF when LV-EF was $\geq 50 \%$ or HFrEF when LV-EF was $<50 \%$ on baseline echocardiography.

\section{Statistical analyses}

Baseline data were presented as mean (standard deviation) for quantitative variables, and absolute number (\%) for frequencies. The $t$ test and Fisher's test were used for comparisons between groups for quantitative variables and frequencies, respectively.

The impact of different comorbidities on NYHA class was assessed by ordinal regression, adjusted for age, sex and $\mathrm{CHF}$ group (HFrEF or HFpEF). In preliminary analyses, the interaction term of the CHF group and comorbidity was included to examine whether the effects of the comorbidity on NYHA class in HFrEF and HFpEF patients should be considered to be of the same or different size. If the $p$ value for interaction was $\geq 0.1$, a common estimate of the odds ratio was presented. In case of a significant $(p<0.05)$ or borderline $(0.05 \leq p<0.1)$ interaction term, homogeneity of the odds ratios was not assumed, and separate estimates in the HFrEF and HFpEF groups were computed.

The association between comorbidities on the SF-36 PF scale was analyzed in the same manner, using linear regression.

Multiple regression analyses (ordinal or linear, respectively) for NYHA class and SF-36 PF were performed, including all comorbidities as covariates for both groups. We also included into these analyses two echocardiographic measures: left ventricular ejection fraction (LV-EF) describing the severity of systolic dysfunction in HFrEF and left ventricular end-diastolic diameter (LVD[ED]) reflecting impaired ventricular filling in HFpEF. This allows weighing the impacts of ventricular dysfunction and comorbidities on symptoms and physical functioning against each other.

Based on the multiple regression models, areas under ROC curves for the prediction of NYHA classes III-IV and explained variances $\left(R^{2}\right)$ for the prediction of the SF-36 physical functioning scale were computed to illustrate the explanatory impact of comorbidities on top of sociodemography and echocardiographic parameters.

\section{Results}

Among 8,368 individuals screened for participation, 4,259 were identified with $\mathrm{CHF}$. Of these, information was missing on LV-EF in 150, NYHA class in 25, both LV-EF and NYHA in 4, and age in 1 patient. Of 4,079 patients available for analysis, 2,785 (68\%) had HFrEF and 1,294
(32\%) had HFpEF. Out of these, 3,476 (85.2\%) completed the SF-36 questionnaire. In multivariate logistic regression analysis including age, sex and CHF group as covariates, only age significantly predicted the completion of the SF-36 questionnaire.

HFpEF patients were older, more frequently female, had higher blood pressures and were less intensively treated with heart failure medications (Table 1). They were also less symptomatic with a lower mean NYHA class and a lower number of CHF symptoms per patient with lower rates for all individual symptoms except peripheral edema (Table 1 and Fig. 1). SF-36 PF score was higher in HFpEF.

Only hypertension and obesity were more frequent in HFpEF, while all other comorbidities were observed more frequently in HFrEF patients. Seven comorbidities did not show an interaction with $\mathrm{CHF}$ group in ordinal regression analysis, i.e., their influence on NYHA class and SF-36 PFS was similar in both groups (Table 2). All these comorbidities had a highly significant impact on patients' symptoms by increasing NYHA class and reducing SF-36 PF score, with COPD and anemia showing the strongest associations. The remaining five comorbidities behaved differently in HFpEF and HFrEF: In HFrEF, hypertension and hyperlipidemia were associated with a better NYHA class, while the latter impacted on the NYHA class negatively in HFpEF and was also associated with a worse SF-36 PF in these patients. CAD had a negative effect on NYHA class in HFpEF only and its association with lower SF-36 PF was significantly stronger than that observed in HFrEF patients. Similarly, a negative impact of PAOD on NYHA class and SF-36 PF was significantly stronger in HFpEF compared with HFrEF. Obesity significantly increased NYHA class in HFpEF only, while there was no significant difference in its (negative) impact on SF-36 PF in both CHF groups.

In multivariate analyses, hyperuricemia, renal dysfunction, anemia, COPD, cerebrovascular disease and atrial fibrillation retained their significantly negative impact on NYHA class in HFrEF, while hypertension and hyperlipidemia retained their protective effect (Fig. 2a). The strength of association for the negatively influencing comorbidities was similar to that of a reduction in LV-EF of $10 \%$ (which corresponds to a quartile of the distribution for this parameter in our cohort), with widely overlapping confidence intervals. In HFpEF, only CAD, anemia, obesity, COPD and atrial fibrillation had a significantly negative effect in multivariate ordinal regression analysis, although wider confidence intervals illustrated the lower statistical power to show influencing factors due to the lower subject number in this group. The effect of these comorbidities appeared to be much stronger than that of an LVD (ED) lowered by $5 \mathrm{~mm}$ (again corresponding to a quartile in our cohort), the most strongly associated 
Table 1 Clinical characteristics

\begin{tabular}{|c|c|c|c|c|}
\hline Table 1 Clinical characteristics & & HFrEF $(n=2,785)$ & HFpEF $(n=1,294)$ & $p$ value \\
\hline & Age (years) & $63 \pm 14$ & $67 \pm 13$ & $<0.001$ \\
\hline & Female sex & $709(25.5 \%)$ & $696(53.8 \%)$ & $<0.001$ \\
\hline & Systolic blood pressure $(\mathrm{mmHg})$ & $123 \pm 20$ & $142 \pm 24$ & $<0.001$ \\
\hline & Diastolic blood pressure $(\mathrm{mmHg})$ & $73 \pm 12$ & $81 \pm 13$ & $<0.001$ \\
\hline & Heart rate $(1 / \mathrm{min})$ & $76 \pm 17$ & $70 \pm 14$ & $<0.001$ \\
\hline & \multicolumn{4}{|l|}{ Medication (n) } \\
\hline & ACEI/ARB & $2,458(88.3 \%)$ & $790(61.1 \%)$ & $<0.001$ \\
\hline & Beta-blocker & $2,352(84.5 \%)$ & $669(51.7 \%)$ & $<0.001$ \\
\hline & Diuretic & $2,213(79.5 \%)$ & $680(52.6 \%)$ & $<0.001$ \\
\hline & Aldosterone antagonist & $1,290(46.3 \%)$ & $61(4.7 \%)$ & $<0.001$ \\
\hline & \multicolumn{4}{|l|}{ Signs and symptoms } \\
\hline & NYHA class & $2.4 \pm 0.6$ & $2.0 \pm 0.6$ & $<0.001$ \\
\hline & SF-36 physical functioning score & $49.6 \pm 28.3$ & $54.4 \pm 27.7$ & $<0.001$ \\
\hline & Dyspnea on exertion & $2,424(87.0 \%)$ & $1,088(84.1 \%)$ & 0.013 \\
\hline & Dyspnea at rest & $327(11.7 \%)$ & $88(6.8 \%)$ & $<0.001$ \\
\hline & Peripheral edema & $713(25.6 \%)$ & $432(33.4 \%)$ & $<0.001$ \\
\hline & Neck vein distention & $187(6.7 \%)$ & $31(2.4 \%)$ & $<0.001$ \\
\hline & Pulmonary rales & $317(11.4 \%)$ & $52(4.0 \%)$ & $<0.001$ \\
\hline & Number of symptoms & $1.4 \pm 1.0$ & $1.3 \pm 0.8$ & $<0.001$ \\
\hline & \multicolumn{4}{|l|}{ Echocardiography } \\
\hline & Left ventricular ejection fraction & $31.2 \pm 9.4$ & $62.0 \pm 8.4$ & n.a.* \\
\hline & End-diastolic LV diameter (mm) & $62.3 \pm 10.1$ & $48.2 \pm 7.6$ & $<0.001$ \\
\hline & \multicolumn{4}{|l|}{ Comorbidities (n) } \\
\hline & Diabetes mellitus & $864(31.0 \%)$ & $313(24.2 \%)$ & $<0.001$ \\
\hline & Hypertension & $1,709(61.4 \%)$ & $1,014(78.4 \%)$ & $<0.001$ \\
\hline & Hyperlipidemia & $1,638(58.8 \%)$ & $699(54.0 \%)$ & 0.004 \\
\hline & Hyperuricemia & $1,086(39.0 \%)$ & $252(19.5 \%)$ & $<0.001$ \\
\hline & Coronary artery disease & $1,286(46.2 \%)$ & $405(31.3 \%)$ & $<0.001$ \\
\hline & Renal dysfunction & $962(34.5 \%)$ & $341(26.4 \%)$ & $<0.001$ \\
\hline & Anemia & $610(21.9 \%)$ & $183(14.1 \%)$ & $<0.001$ \\
\hline & Obesity & $746(26.8 \%)$ & $486(37.6 \%)$ & $<0.001$ \\
\hline & COPD & $421(15.1 \%)$ & $163(12.6 \%)$ & 0.035 \\
\hline & Peripheral artery disease & $292(10.5 \%)$ & $98(7.6 \%)$ & $<0.001$ \\
\hline & Cerebrovascular disease & $308(11.1 \%)$ & $117(9.0 \%)$ & $<0.001$ \\
\hline & Atrial fibrillation & $581(20.9 \%)$ & $124(9.6 \%)$ & $<0.001$ \\
\hline * Different by definition of & Number of comorbidities & $3.8 \pm 2.1$ & $3.2 \pm 1.9$ & $<0.001$ \\
\hline
\end{tabular}

parameter on routine echocardiography in this patient group. SF-36 PF score was negatively affected in HFrEF by all comorbidities except for diabetes, hypertension and hyperlipidemia, with the last two again showing a protective effect with regard to this parameter (Fig. 2b). The effects tended to be stronger than that of an LV-EF reduction. In $\mathrm{HFpEF}$, hyperuricemia, CAD, renal dysfunction, anemia, obesity, COPD, PAOD and atrial fibrillation were associated with lower SF-36 PF score. Again, the effect of reduced LVD (ED) was significant but weak in comparison with comorbidities. LV-EF had no significant effect on HFpEF and the same held true for LVD (ED) in $\mathrm{HFrEF}$.
Accordingly, adding both echocardiographic variables as covariates improved the prediction of NYHA classes III or IV in HFrEF, derived from the multivariate regression model, while AUC remained virtually unchanged in HFpEF (Fig. 3a). Expansion of the set of covariates for all comorbidities further improved the AUC in HFrEF to an extent similar to that provided by the addition of the echocardiographic variables. However, in HFpEF, the increase in AUC was much larger (+0.100 vs. +0.029), resulting in a similar AUC as in HFrEF for the final multivariate model that included all covariates, with a relatively larger share supplied by comorbidities in HFpEF. 
Fig. 1 Distribution across a NYHA grades and b individual number of $\mathrm{CHF}$ symptoms in patients with HFrEF (open columns) or HFpEF (filled columns)
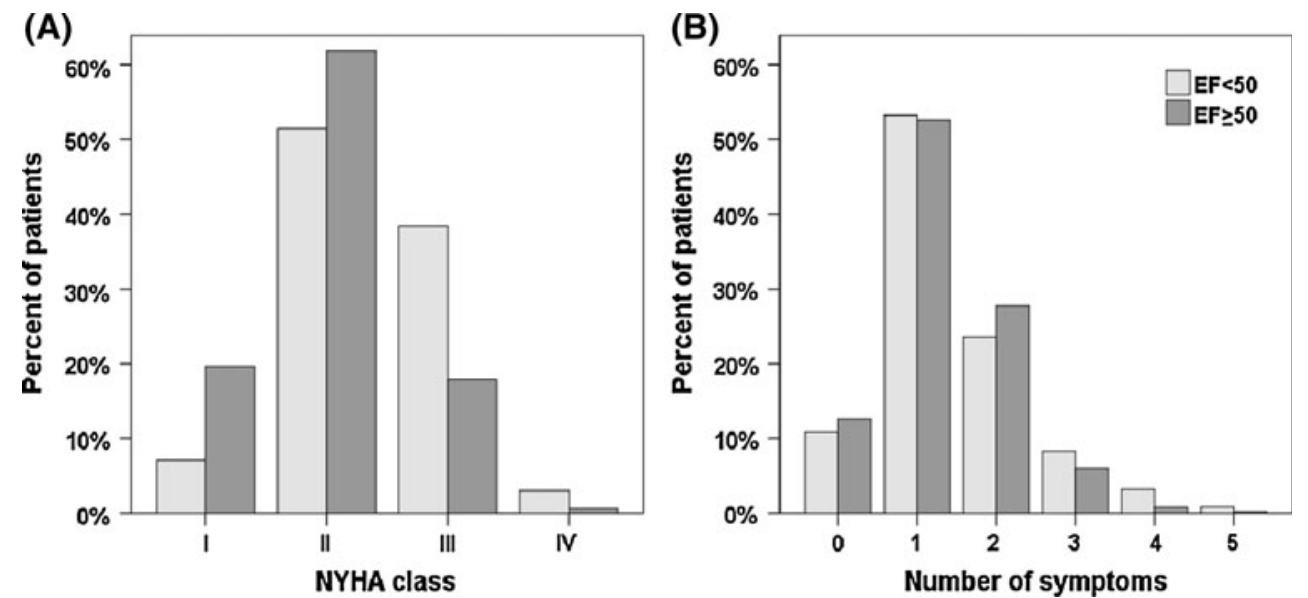

In $\mathrm{HFpEF}$, considerably more of the overall variability in SF-36 PF score was explained by sex and age only compared with HFrEF (Fig. 3b). Again, adding echocardiographic variables did not improve the multivariate model in HFpEF, while some improvement was noted for $\mathrm{HFrEF}$, and the inclusion of all comorbidities further improved $r^{2}$ by $7.6 \%$. In HFpEF, comorbidities added $12.1 \%$ and thereby approximately doubled the explained variance, resulting in an $r^{2}$ of $25.1 \%$. The variance explained by comorbidities was therefore 1.59 -fold higher in HFpEF than in HFrEF. The overall explained variance of SF-36 PF in HFpEF by sex, age, echocardiographic variables and comorbidities was 1.49-fold larger than in HFrEF, even though the echocardiographic variables had no impact on $r^{2}$ in HFpEF.

\section{Discussion}

To our knowledge, our analysis is the first to evaluate the relative impact of multiple comorbidities on physical function in CHF in general, and in HFpEF and HFrEF in particular.

We find that patients with HFpEF are significantly less symptomatic than those with HFrEF. Of this lower CHF symptom load in absolute terms, a relatively larger part is explained by comorbidities, leaving a smaller fraction to be explained by heart failure itself (or possibly other factors). This is in spite of higher frequencies for most of the major comorbidities investigated and a higher number of comorbidities per patient in HFrEF.

CHF is a disease primarily of the elderly [1]; comorbidities are prevalent in these patients [4] and will be more so in the future due to an aging population [15]. At the same time, specialization and subspecialization continues to be on the rise $[16,17]$, resulting in a strong focus of different specialists on individual diseases from their respective area of expertise, which may in turn compromise the care for other comorbidities [18]. It has previously been shown that comorbidities have an impact on hospitalizations and mortality in patients with CHF [4, 19]. Our data complement this report, as we find a significant impact on patients' physical impairment in a large and diverse CHF population from observational as well as interventional trials with a harmonized basic data set. Comorbidities are therefore associated with relevant suffering in daily life in patients with CHF. At the same time, they strongly influence the main symptom that leads to a CHF-oriented diagnostic workup and is used to judge the course of disease and success of physicians' interventions. This observation may appear to be obvious and intuitive. However, if that is so, the above-mentioned trends in the organization of medical care might consequentially be considered suboptimal and counterintuitive.

All individual comorbidities had a significant impact on physical impairment in at least one of both heart failure groups. Several of these (such as COPD, history of stroke, atrial fibrillation, anemia or diabetes) generally limit exercise capacity by themselves, even in patients without CHF [20-23]. Others (like renal dysfunction or hyperuricemia) may in fact rather be indicators of more advanced and therefore more symptomatic CHF [24, 25]. Similarly, the association of hypertension and hyperlipidemia with a lower NYHA class in HFrEF may be due to the phenomenon of reverse epidemiology, i.e., the association of low blood pressure and lower lipid levels with an adverse prognosis and more advanced disease [26]. Such an effect was not detectable for hypertension in HFpEF, and hyperlipidemia actually worsened NYHA class and SF-36 $\mathrm{PF}$ score. This differential impact on physical function contrasts with a recent report that showed a similar impact of hyperlipidemia and hypertension on prognosis in HFrEF as compared to HFpEF. Further studies will be needed to better understand the associations of comorbidities with 
Table 2 Impact on NYHA class and SF-36 physical functioning scale of comorbidities, corrected for age and sex
Separate estimates are given for HFrEF and HFpEF subgroups when the $p$ value for interaction of comorbidity and ventricular function is significant $(<0.05)$ or borderline $(<0.1)$

\begin{tabular}{|c|c|c|c|c|}
\hline \multirow[t]{2}{*}{ Comorbidity } & \multicolumn{2}{|l|}{ Impact on NYHA class } & \multicolumn{2}{|l|}{ Impact on SF-36 PFS } \\
\hline & Odds ratio $(95 \% \mathrm{CI})$ & $p$ value & Coefficient $(95 \%$ CI $)$ & $p$ value \\
\hline \multicolumn{5}{|c|}{ Comorbidities without significant interaction } \\
\hline \multicolumn{5}{|l|}{ COPD } \\
\hline Interaction & & 0.18 & & 0.10 \\
\hline Common estimate & $1.82(1.54 ; 2.15)$ & $<0.001$ & $-12.79(-10.27 ;-15.30)$ & $<0.001$ \\
\hline \multicolumn{5}{|c|}{ Cerebrovascular disease } \\
\hline Interaction & & 1.00 & & 0.43 \\
\hline Common estimate & 1.49 (1.26; 1.77) & $<0.001$ & $-8.27(-5.35 ;-11.18)$ & $<0.001$ \\
\hline \multicolumn{5}{|l|}{ Atrial fibrillation } \\
\hline Interaction & & 0.10 & & 0.73 \\
\hline Common estimate & $1.59(1.37 ; 1.83)$ & $<0.001$ & $-6.14(-3.74 ;-8.55)$ & $<0.001$ \\
\hline \multicolumn{5}{|l|}{ Diabetes } \\
\hline Interaction & & 0.79 & & 0.09 \\
\hline Common estimate & $1.26(1.11 ; 1.45)$ & $<0.001$ & $-5.82(-3.82 ;-7.83)$ & $<0.001$ \\
\hline \multicolumn{5}{|l|}{ Renal dysfunction } \\
\hline Interaction & & 0.60 & & 0.16 \\
\hline Common estimate & $1.51(1.31 ; 1.73)$ & $<0.001$ & $-7.41(-5.38 ;-9.44)$ & $<0.001$ \\
\hline \multicolumn{5}{|l|}{ Anemia } \\
\hline Interaction & & 0.36 & & 0.25 \\
\hline Common estimate & $1.77(1.51 ; 2.07)$ & $<0.001$ & $-9.74(-7.46 ;-12.02)$ & $<0.001$ \\
\hline \multicolumn{5}{|l|}{ Hyperuricemia } \\
\hline Interaction & & 0.58 & & 0.10 \\
\hline Common estimate & $1.60(1.40 ; 1.82)$ & $<0.001$ & $-6.53(-4.61 ; 8.45)$ & $<0.001$ \\
\hline \multicolumn{5}{|c|}{ Comorbidities with significant interaction } \\
\hline \multicolumn{5}{|l|}{ Obesity } \\
\hline Interaction & & 0.01 & & 0.68 \\
\hline HFrEF subgroup & $1.10(0.93 ; 1.30)$ & 0.26 & & \\
\hline HFpEF subgroup & $1.53(1.22 ; 1.92)$ & $<0.001$ & & \\
\hline Common estimate & & & $-4.35(-2.39 ;-6.30)$ & $<0.001$ \\
\hline \multicolumn{5}{|l|}{ Hypertension } \\
\hline Interaction & & 0.06 & & 0.02 \\
\hline HFrEF subgroup & $0.75(0.64 ; 0.88)$ & 0.001 & $0.28(-2.09 ; 2.66)$ & 0.82 \\
\hline HFpEF subgroup & $0.89(0.66 ; 1.18)$ & 0.41 & $-4.6(-0.65 ;-8.61)$ & 0.02 \\
\hline \multicolumn{5}{|l|}{ Hyperlipidemia } \\
\hline Interaction & & $<0.001$ & & $<0.001$ \\
\hline HFrEF subgroup & $0.75(0.65 ; 0.87)$ & $<0.001$ & $1.82(-0.41 ; 4.04)$ & 0.11 \\
\hline HFpEF subgroup & $1.38(1.11 ; 1.73)$ & 0.005 & $-6.28(3.16 ; 9.40)$ & $<0.001$ \\
\hline \multicolumn{5}{|c|}{ Coronary artery disease } \\
\hline Interaction & & 0.001 & & 0.001 \\
\hline HFrEF subgroup & $0.94(0.80 ; 1.10)$ & 0.40 & $-5.62(-3.24 ;-7.99)$ & $<0.001$ \\
\hline HFpEF subgroup & $1.71(1.33 ; 2.19)$ & $<0.001$ & $-12.01(-8.59 ;-15.42)$ & $<0.001$ \\
\hline \multicolumn{5}{|c|}{ Peripheral artery disease } \\
\hline Interaction & & 0.02 & & 0.04 \\
\hline HFrEF subgroup & $1.23(0.97 ; 1.56)$ & 0.083 & $-10.70(-7.14 ;-14.26)$ & $<0.001$ \\
\hline HFpEF subgroup & $1.94(1.28 ; 2.93)$ & 0.002 & $-17.73(-11.94 ;-23.52)$ & $<0.001$ \\
\hline
\end{tabular}

symptom burden and prognosis in HFpEF patients [26]. Hypertension may have a stronger negative impact on HFpEF due to ventriculo-vascular coupling [27] and because it is a key factor for the development of left ventricular hypertrophy [28] and diastolic dysfunction [29]. Alternatively, the reverse association of low blood 

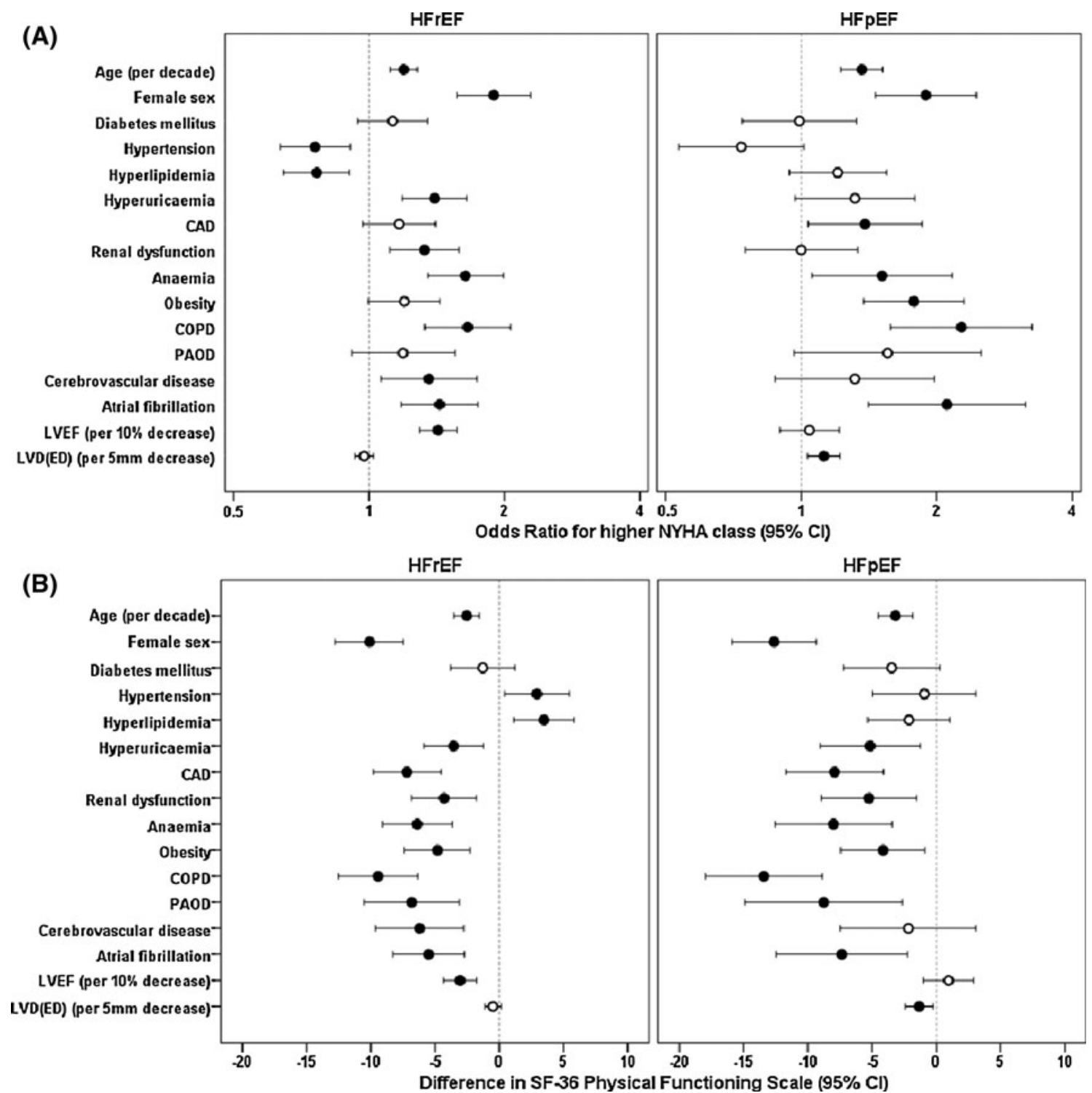

Fig. 2 Odds ratios in multivariate analyses for a higher NYHA class and b SF-36 physical functioning score in HFrEF or HFpEF

pressures with advanced disease may be less pronounced in $\mathrm{HFpEF}$, an explanation that would also be in concordance with the overall lower symptom load, indicating a less advanced disease stage in our HFpEF group. This alternative explanation could also account for the differential impact of obesity. While this comorbidity would be expected to lead to worse physical functioning, as observed in our HFpEF group, this effect has likely been offset by reverse epidemiology in the HFrEF group, leading to a neutral impact on the NYHA class. The more pronounced influence of PAOD may be indicative of the relevance of ventriculo-vascular coupling for the development of HFpEF [27, 30].

Why should the overall effect of comorbidities on physical function be greater in HFpEF than in HFrEF patients, as observed in our analysis?
Patients with $\mathrm{HFpEF}$ are a heterogeneous group. While the presence of a lowered LV-EF adds some cardio-specificity to the clinical diagnosis of $\mathrm{CHF}$, its absence does not. HFpEF therefore by principle will be much less specific for cardiac abnormalities as the underlying pathology for the clinical picture of heart failure. Consequentially, basing HFpEF exclusively on the clinical picture and the absence of major abnormalities in systolic function will lead to a higher number of cases falsely attributed to cardiac abnormalities [31]. It is likely that some of these cases will have non-cardiac comorbidities as a main reason for reduced physical function.

It is also possible that comorbidities have a relatively higher impact on physical impairment in patients with true $\mathrm{CHF}$ due to cardiac (mainly diastolic) dysfunction with normal ejection fraction. This would suggest that diastolic 
Fig. 3 a AUC to predict higher NYHA class and $\mathbf{b} r^{2}$ of multivariate linear model for SF-36 physical functioning score according to the set of covariates used for model building
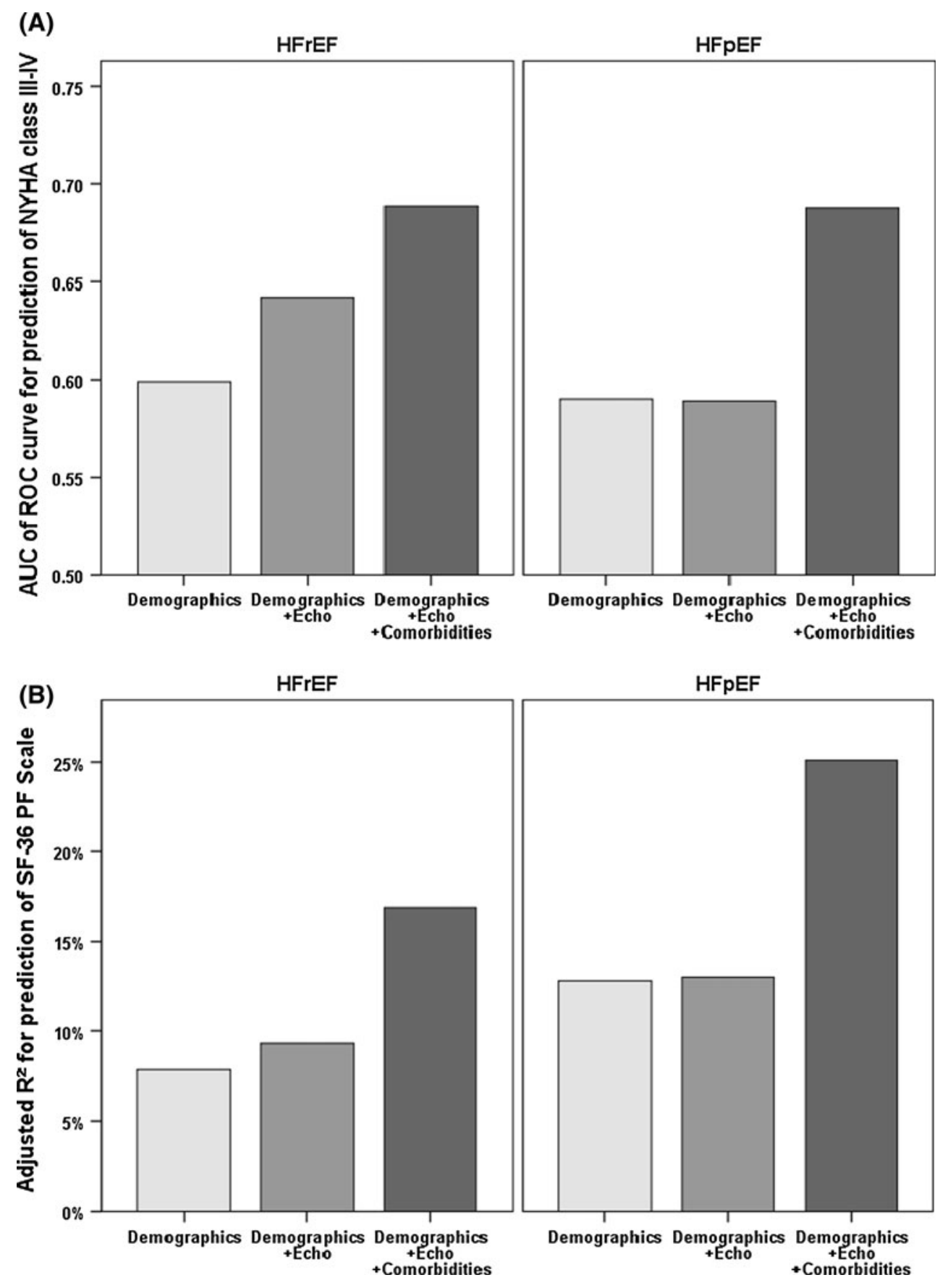

dysfunction by itself would have a smaller effect on physical function than relevant systolic dysfunction (which is usually accompanied by some degree of diastolic function and in that sense is additive). Although the similarities in morbidity and mortality [6, 7] as well as health-care utilization [32] between HFpEF and HFrEF may seem to argue against this, adverse prognosis in HFpEF has been reported to be associated in a large part with non-CHF events [8-10] and a differential influence of gender has been described [33]. Further supporting this hypothesis is the fact that the echocardiographic variable most strongly associated with physical function in each group had a much stronger effect in HFrEF than in HFpEF. Cardiac abnormalities in HFpEF may therefore bear a smaller part of adverse prognosis and, as we show here, of impairment in physical function.

Our results strongly support two major calls to researchers and physicians dealing with heart failure patients: Firstly, a diagnosis of HFpEF must not be based on the absence of systolic abnormalities, but rather on the 
presence of non-systolic cardiac abnormalities. Otherwise, HFpEF populations will by conception include a larger number of non-cardiac etiologies for impaired physical function, making clinical research aimed at specific treatments for HFpEF challenging (and some would argue, impossible) [31, 34].

Secondly, caring for patients with CHF must include a strong focus on the diagnosis and treatment of comorbidities, because patients' suffering can only be partly addressed by treating their cardiac abnormalities only.

Some limitations of our analysis have to be addressed. Although we were limited in our analysis to a certain set of comorbidities, several other diseases that were not assessed in all of the studies included may also have an impact on physical function in heart failure patients (e.g., sleep-disordered breathing, depression, cognitive dysfunction, thyroid disorders, osteoporosis). We only had a basic set of echocardiographic data to include in our analysis. Including more recent markers of diastolic function might well have shown a stronger effect on physical function. However, smaller left ventricular end-diastolic dimensions have been shown to be characteristic of patients with $\mathrm{HFpEF}$ [27, 35] and were significantly associated with lower NYHA class in our cohort. Some of the evidence we present is of an indirect nature. A direct estimate of the impact of HFrEF or $\mathrm{HFpEF}$ itself on physical function in comparison with a non-CHF population would be most interesting, but cannot be derived from our data set. The diagnosis of comorbidities was largely history based and therefore suboptimal. However, we assume that such an approach will lead to under- rather than overdiagnosis and more cases of e.g., COPD, CAD or (paroxysmal) atrial fibrillation would have been revealed by more intensive diagnostic testing, increasing the relative impact of comorbidities on physical function. Similarly, a confirmation of our findings in an unselected patient population will be necessary. Because such a population will be older and more comorbid, a different impact of comorbidities cannot be excluded.

\section{Conclusion}

Comorbidities have a significant impact on NYHA class and SF-36 PF in HFrEF as well as in HFpEF. The influence of individual comorbidities is different in HFrEF as compared to HFpEF. The overall impact of comorbidities is higher in HFpEF than in HFrEF. This implies that comorbidities should play a larger role in the differential diagnosis of patients with exertional dyspnea and preserved ejection fraction. Focusing treatment on comorbidities may be more beneficial in HFpEF than in HFrEF.
Acknowledgments This study was supported by grants from the German Federal Ministry of Education and Research (Competence Network Heart Failure, TP 7 (FKZ 01GI0205), including a scholarship to Raoul Stahrenberg) and clinical trial program Aldo-DHF (FKZ 01KG0506)). Götz Gelbrich (project biometrician for CNHF) had full access to all of the data in the study and takes responsibility for the integrity of the data and the accuracy of the data analysis.

\section{Conflict of interest None declared.}

Open Access This article is distributed under the terms of the Creative Commons Attribution Noncommercial License which permits any noncommercial use, distribution, and reproduction in any medium, provided the original author(s) and source are credited.

\section{References}

1. Lloyd-Jones D, Adams RJ, Brown TM, Carnethon M, Dai S, De Simone G et al (2010) Heart disease and stroke statistics-2010 update: a report from the American Heart Association. Circulation 121(7):e46-e215

2. Kitzman DW, Groban L (2008) Exercise intolerance. Heart Fail Clin 4(1):99-115

3. Krum H, Gilbert RE (2003) Demographics and concomitant disorders in heart failure. Lancet 362(9378):147-158

4. Braunstein JB, Anderson GF, Gerstenblith G, Weller W, Niefeld M, Herbert R et al (2003) Noncardiac comorbidity increases preventable hospitalizations and mortality among Medicare beneficiaries with chronic heart failure. J Am Coll Cardiol 42(7):1226-1233

5. Kitzman DW, Little WC, Brubaker PH, Anderson RT, Hundley WG, Marburger CT et al (2002) Pathophysiological characterization of isolated diastolic heart failure in comparison to systolic heart failure. JAMA 288(17):2144-2150

6. Owan TE, Hodge DO, Herges RM, Jacobsen SJ, Roger VL, Redfield MM (2006) Trends in prevalence and outcome of heart failure with preserved ejection fraction. $\mathrm{N}$ Engl $\mathrm{J}$ Med 355(3):251-259. doi:10.1056/NEJMoa052256

7. Lee DS, Gona P, Vasan RS, Larson MG, Benjamin EJ, Wang TJ et al (2009) Relation of Disease Pathogenesis and Risk Factors to Heart Failure With Preserved or Reduced Ejection Fraction: Insights From the Framingham Heart Study of the National Heart, Lung, and Blood Institute. Circulation 119(24):3070-3077. doi: 10.1161/CIRCULATIONAHA.108.815944

8. Ahmed A, Rich MW, Fleg JL, Zile MR, Young JB, Kitzman DW et al (2006) Effects of digoxin on morbidity and mortality in diastolic heart failure: the Ancillary Digitalis Investigation Group Trial. Circulation 114(5):397-403

9. Yusuf S, Pfeffer MA, Swedberg K, Granger CB, Held P, McMurray JJ et al (2003) Effects of candesartan in patients with chronic heart failure and preserved left-ventricular ejection fraction: the CHARM-Preserved Trial. Lancet 362(9386):777-781

10. Perez de Isla L, Canadas V, Contreras L, Almeria C, Rodrigo JL, Aubele AL et al (2009) Diastolic heart failure in the elderly: inhospital and long-term outcome after the first episode. Int $\mathbf{J}$ Cardiol 134(2):265-270

11. Shah SJ, Gheorghiade M (2008) Heart failure with preserved ejection fraction: treat now by treating comorbidities. JAMA 300(4):431-433

12. Mehrhof F, Loffler M, Gelbrich G, Ozcelik C, Posch M, Hense HW, et al. (2009 Aug 11) A network against failing heartsIntroducing the German "Competence Network Heart Failure". Int J Cardiol 
13. Levey AS, Bosch JP, Lewis JB, Greene T, Rogers N, Roth D (1999) A more accurate method to estimate glomerular filtration rate from serum creatinine: a new prediction equation. Modification of Diet in Renal Disease Study Group. Ann Intern Med 130(6):461-470

14. Ware JE Jr, Sherbourne CD (1992) The MOS 36-item short-form health survey (SF-36). I. Conceptual framework and item selection. Med Care 30(6):473-483

15. Kinsella KaWH, U.S. Census Bureau. P95/09-1, An Aging World: 2008. International Population Reports. Washington, DC: U.S. Government Printing Office; 2009

16. Lakhan SE, Laird C (2009) Addressing the primary care physician shortage in an evolving medical workforce. Int Arch Med 2(1): 14

17. Primary Care Professionals: Recent Supply Trends, Projections, and Valuation of Services: United States Government Accountability Office (2008)

18. Redelmeier DA, Tan SH, Booth GL (1998) The treatment of unrelated disorders in patients with chronic medical diseases. N Engl J Med 338(21):1516-1520

19. von Haehling S, Schefold JC, Hodoscek LM, Doehner W, Mannaa M, Anker SD et al (2010) Anaemia is an independent predictor of death in patients hospitalized for acute heart failure. Clin Res Cardiol 99(2):107-113

20. Luderitz B, Jung W (2000) Quality of life in patients with atrial fibrillation. Arch Intern Med 160(12):1749-1757

21. Odden MC, Whooley MA, Shlipak MG (2004) Association of chronic kidney disease and anemia with physical capacity: the heart and soul study. J Am Soc Nephrol 15(11):2908-2915

22. O'Donnell DE, Laveneziana P (2007) Dyspnea and activity limitation in COPD: mechanical factors. COPD 4(3):225-236

23. Saraiva RM, Duarte DM, Duarte MP, Martins AF, Poltronieri AV, Ferreira ME et al (2005) Tissue Doppler imaging identifies asymptomatic normotensive diabetics with diastolic dysfunction and reduced exercise tolerance. Echocardiography 22(7):561-570

24. Ronco C, Haapio M, House AA, Anavekar N, Bellomo R (2008) Cardiorenal syndrome. J Am Coll Cardiol 52(19):1527-1539

25. Anker SD, Doehner W, Rauchhaus M, Sharma R, Francis D, Knosalla $C$ et al (2003) Uric acid and survival in chronic heart failure: validation and application in metabolic, functional, and hemodynamic staging. Circulation 107(15):1991-1997
26. Guder G, Frantz S, Bauersachs J, Allolio B, Wanner C, Koller MT et al (2009) Reverse epidemiology in systolic and nonsystolic heart failure: cumulative prognostic benefit of classical cardiovascular risk factors. Circ Heart Fail 2(6):563-571

27. Lam CS, Roger VL, Rodeheffer RJ, Bursi F, Borlaug BA, Ommen SR et al (2007) Cardiac structure and ventricular-vascular function in persons with heart failure and preserved ejection fraction from Olmsted County, Minnesota. Circulation 115(15):1982-1990

28. Levy D, Anderson KM, Savage DD, Kannel WB, Christiansen JC, Castelli WP (1988) Echocardiographically detected left ventricular hypertrophy: prevalence and risk factors. The Framingham Heart Study. Ann Intern Med 108(1):7-13

29. Dwyer EM, Asif M, Ippolito T, Gillespie M (2000) Role of hypertension, diabetes, obesity, and race in the development of symptomatic myocardial dysfunction in a predominantly minority population with normal coronary arteries. Am Heart J 139(2 Pt 1):297-304

30. Correa de Sa DD, Hodge DO, Slusser JP, Redfield MM, Simari RD, Burnett JC et al (2010) Progression of preclinical diastolic dysfunction to the development of symptoms. Heart 96(7):528-532

31. Paulus WJ, van Ballegoij JJ (2010) Treatment of heart failure with normal ejection fraction: an inconvenient truth!. J Am Coll Cardiol 55(6):526-537

32. Liao L, Jollis JG, Anstrom KJ, Whellan DJ, Kitzman DW, Aurigemma GP et al (2006) Costs for heart failure with normal vs reduced ejection fraction. Arch Intern Med 166(1):112-118

33. Alla F, Al-Hindi AY, Lee CR, Schwartz TA, Patterson JH, Adams KF Jr (2007) Relation of sex to morbidity and mortality in patients with heart failure and reduced or preserved left ventricular ejection fraction. Am Heart J 153(6):1074-1080

34. Kamp O, Metra M, De Keulenaer GW, Pieske B, Conraads V, Zamorano J et al (2010) Effect of the long-term administration of nebivolol on clinical symptoms, exercise capacity and left ventricular function in patients with heart failure and preserved left ventricular ejection fraction: background, aims and design of the ELANDD study. Clin Res Cardiol 99(2):75-82

35. Bursi F, Weston SA, Redfield MM, Jacobsen SJ, Pakhomov S, Nkomo VT et al (2006) Systolic and diastolic heart failure in the community. JAMA 296(18):2209-2216 